\title{
Publisher Correction: No hot and luminous progenitor for Tycho's supernova
}

\section{T. E. Woods, P. Ghavamian, C. Badenes and M. Gilfanov}

Correction to: Nature Astronomy https://doi.org/10.1038/s41550-017-0263-5; published online 25 September 2017.

In the version of this Article originally published the variable in equation (1) representing neutral hydrogen was incorrect and should have read $\mathrm{H}^{0}$. This has been corrected in all versions of the Article. 